\title{
ON THE ESSENTIAL MAXIMALITY OF LINEAR OPERATORS IN A HILBERT SPACE
}

\author{
Bernhard Steinig
}

\section{Introduction}

In this paper we want to define and study the essential maximality of a linear (not necessarily bounded) operator in a Hilbert space. The concept of essential maximality is a generalization of the essential self-adjointness of a symmetric operator in the sense that an essentially maximal symmetric operator is essentially self-adjoint. To the author's knowledge, the essential maximality has been previously defined and studied only by R.A. Goldstein [1], P. Hess [2], and J. Tervo [7] for linear partial differential operators (cf. also I.S. Louhivaara and C.G. Simader [4]; for a survey of results on the essential self-adjointness of differential operators of mathematical physics we refer to H. Kalf, U.-W. Schmincke, J. Walter and R. Wüst [3]).

In 1 we shall define the essential maximality of a linear operator in view of a second one. We also give a method for verifying the essential maximality of given operators. From a corollary of this result it follows that the methods developed in the literature for the verification of the essential self-adjointness of symmetric operators can be applied to the proof of the essential maximality of linear operators.

In 2 the methods of 1 will be applied for a tensor product of linear operators, and we shall prove the relation

$$
\left(T_{1} \otimes T_{2}\right)^{*}=\overline{T_{1}^{*} \otimes T_{2}^{*}}
$$

for two densely defined closable operators $T_{1}$ and $T_{2}$; in this relation the right side means the closure of the tensor product $T_{1}^{*} \otimes T_{2}^{*}$ in the Hilbert space $H_{1} \widehat{\otimes} H_{2}$ which is defined as the completion of the tensor product $H_{1} \otimes H_{2}$. K. Vala [8] has proven this result in the case of bounded operators, and he has also showed $T_{1}^{*} \otimes T_{2}^{*} \subset\left(T_{1} \otimes T_{2}\right)^{*}$ for general linear operators. The result is also known at least for unbounded self-adjoint operators; cf. J. Weidmann [9], pp. 259-268, who has proved the result using the spectral theorem. We do not use the spectral theorem in our considerations.

In 3 the results of 1 and 2 will be formulated for linear partial differential operators. 
On this occasion I would like to express my gratitude to Professor I.S. Louhivaara of Freie Universität Berlin for his interest and his extensive support during my work on the manuscript. I also want to thank Professor R. Wüst of Technische Universität Berlin for his valuable suggestions which clarified the ideas of this paper.

\section{A characterization of the essential maximality of linear operators in a Hilbert space}

Let $T$ and $T^{\prime}$ be two densely defined linear operators in the complex Hilbert space $H$ (with the domains $D(T)$ and $D\left(T^{\prime}\right)$ ) such that the relation

$$
(T \varphi, \psi)=\left(\varphi, T^{\prime} \psi\right)
$$

is valid for all $\varphi \in D(T)$ and all $\psi \in D\left(T^{\prime}\right)$ (i.e. the operators $T$ and $T^{\prime}$ are formal adjoints of each other). The operators $T$ and $T^{\prime}$ are closable, because the adjoint operators $T^{*}$ and $T^{\prime *}$ of $T$ and $T^{\prime}$ in $H$ are densely defined (e.g. $\left.T^{\prime} \subset T^{*}\right)$, and we have the closures $\bar{T}=T^{* *}$ and $\overline{T^{\prime}}=T^{* *}$.

We say that the operator $T$ essentially maximal in view of the operator $T^{\prime}$ if the relation

$$
T^{*}=\overline{T^{\prime}}
$$

is valid. Of course, in this case also $T^{\prime}$ is essentially maximal in view of $T$ (since the above relation implies $\bar{T}=T^{\prime *}$ ).

A closable densely defined operator $T$ in a Hilbert space is of course essentially maximal in view of the adjoint operator $T^{*}$.

If the relation $\bar{T}=T^{*}$ holds for a symmetric operator $T$, we call $T$ essentially self-adjoint.

We shall refer to the following result several times (for the proof cf. e.g. F. Riesz and B. Sz.-Nagy [5], pp. 322-323):

Theorem 1.1. Let $H$ be a complex Hilbert space. Let $T: D(T) \rightarrow H$, $D(T) \subset H$, be a densely defined closed linear operator. Then the linear operator $T^{*} T+I$

$$
\begin{gathered}
\left(T^{*} T+I\right): D\left(T^{*} T\right) \rightarrow H, \\
D\left(T^{*} T\right):=\left\{x \in D(T) \mid T x \in D\left(T^{*}\right)\right\},
\end{gathered}
$$

is densely defined, bijective and self-adjoint.

Theorem 1.2. Let $T$ and $T^{\prime}$ be two densely defined operators in a Hilbert space $H$ with the dense domains $D(T)$ and $D\left(T^{\prime}\right)$ so that $T$ and $T^{\prime}$ are formal adjoints to each other. Then the operator $T$ is essentially maximal in view of $T^{\prime}$ if and only if the condition

$$
R\left(\overline{T^{\prime}} \bar{T}+I\right)=H
$$

is satisfied. 
Proof. A. Let $T$ be essentially maximal in view of $T^{\prime}$. Then by Theorem 1.1 one has

$$
R\left(\overline{T^{\prime}} \bar{T}+I\right)=R\left(T^{*} \bar{T}+I\right)=H .
$$

B. Let now $R\left(\overline{T^{\prime}} \bar{T}+I\right)=H$. Because $\overline{T^{\prime}} \subset T^{*}$, one has

$$
\overline{T^{\prime}} \bar{T}+I \subset T^{*} \bar{T}+I .
$$

Since by Theorem 1.1 the operator $\left(T^{*} \bar{T}+I\right): D\left(T^{*} \bar{T}\right) \rightarrow H$ is bijective and $R\left(\overline{T^{\prime}} \bar{T}+I\right)=H$, it follows that

$$
\overline{T^{\prime}} \bar{T}+I=T^{*} \bar{T}+I
$$

and consequently

$$
\overline{T^{\prime}} \bar{T}=T^{*} \bar{T} .
$$

Because of the self-adjointness of $T^{*} \bar{T}$ the operator $\overline{T^{\prime}} \bar{T}$ is densely defined and we have

$$
\left(\overline{T^{\prime}} \bar{T}\right)^{*}=\overline{T^{\prime}} \bar{T} .
$$

From the relation

$$
\overline{T^{\prime}} \bar{T} \subset T^{*} T^{\prime *} \subset\left(\overline{T^{\prime}} \bar{T}\right)^{*}=\overline{T^{\prime}} \bar{T}
$$

we get

$$
\overline{T^{\prime}} \bar{T}=T^{*} T^{*} .
$$

Take $y \in D\left(T^{*}\right)$. By Theorem 1.1 there exists an element $v \in D\left(T^{\prime *} \overline{T^{\prime}}\right)$ with

$$
y=\left(T^{*} \overline{T^{\prime}}+I\right) v
$$

which means

$$
y-v=T^{\prime *} \overline{T^{\prime}} v .
$$

Because $D\left(\overline{T^{\prime}}\right) \subset D\left(T^{*}\right)$, the difference $y-v$ is an element of $D\left(T^{*}\right)$, and we have by (1.1) the following equations

$$
\begin{aligned}
T^{*}(y-v) & =T^{*}\left(T^{\prime *} \overline{T^{\prime}} v\right) \\
& =\left(T^{*} T^{\prime *}\right)\left(\overline{T^{\prime}} v\right) \\
& =\left(\overline{T^{\prime}} \bar{T}\right)\left(\overline{T^{\prime}} v\right) \\
& =\overline{T^{\prime}}\left(\bar{T} \overline{T^{\prime}} v\right) .
\end{aligned}
$$

Since one has $D(\bar{T}) \subset D\left(T^{\prime *}\right)$, we get

$$
T^{*}(y-v)=\overline{T^{\prime}}\left(T^{\prime *} \overline{T^{\prime}} v\right)=\overline{T^{\prime}}(y-v) .
$$


Therefore the difference $y-v$ is an element of $D\left(\overline{T^{\prime}}\right)$. Because $v \in D\left(\overline{T^{\prime}}\right)$, it follows $y \in D\left(\overline{T^{\prime}}\right)$ and one has $D\left(T^{*}\right) \subset D\left(\overline{T^{\prime}}\right)$. Thus we have shown

$$
D\left(T^{*}\right)=D\left(\overline{T^{\prime}}\right) \quad \text { and } \quad T^{*}=\overline{T^{\prime}} .
$$

Corollary 1.3. Let $T$ and $T^{\prime}$ be two densely defined operators, formal adjoints to each other, in a Hilbert space $H$ as in Theorem 1.2.

Each of the following conditions implies the essential maximality:

(i) $\overline{R\left(T^{\prime} T+I\right)}=H$,

(ii) $T^{\prime} T$ is essentially self-adjoint.

Thereby the domain $D\left(T^{\prime} T\right)$ is defined by

$$
D\left(T^{\prime} T\right):=\left\{x \in D(T) \mid T x \in D\left(T^{\prime}\right)\right\} .
$$

Proof. In both cases one can easily show that the relation

$$
R\left(\overline{T^{\prime}} \bar{T}+I\right)=H
$$

is valid ([6], p. 19).

\section{The adjoint operator of the tensor product of operators}

For two complex Hilbert spaces $H_{1}$ and $H_{2}$ one defines the (algebraic) tensor product $H_{1} \otimes H_{2}$ equipped with the usual scalar product. The completion of $H_{1} \otimes H_{2}$ in the topology induced by this scalar product will be denoted by $H_{1} \widehat{\otimes} H_{2}$. The tensor product $T_{1} \otimes T_{2}$ of two operators $T_{1}, T_{2}\left(T_{j}: D\left(T_{j}\right) \rightarrow H_{j}, j=1,2\right)$ is defined as an operator in $H_{1} \widehat{\otimes} H_{2}$ by

$$
D\left(T_{1} \otimes T_{2}\right):=D\left(T_{1}\right) \otimes D\left(T_{2}\right)
$$

and

$$
\left(T_{1} \otimes T_{2}\right)\left(x_{1} \otimes x_{2}\right):=T_{1} x_{1} \otimes T_{2} x_{2} \quad \text { for } x_{j} \in D\left(T_{j}\right) .
$$

For the complete definitions we refer to J. Weidmann [9], pp. 47-49, 259-268.

We shall prove the following theorem.

Theorem 2.1. Let $H_{1}$ and $H_{2}$ be two Hilbert spaces. By $T_{j}$ we denote a densely defined closable linear operator from $D\left(T_{j}\right)\left(\subset H_{j}\right)$ into $H_{j}(j=1,2)$. Then the operator $T_{1} \otimes T_{2}$ in the Hilbert space $H_{1} \widehat{\otimes} H_{2}$ is essentially maximal in view of the operator $T_{1}^{*} \otimes T_{2}^{*}$ :

$$
\left(T_{1} \otimes T_{2}\right)^{*}=\overline{T_{1}^{*} \otimes T_{2}^{*}} .
$$

Remark 2.2. (i) The statement of the theorem is reasonable since by a direct use of the definition of the tensor product of operators we have

$$
\left(\left(T_{1} \otimes T_{2}\right) \varphi, \psi\right)_{H_{1} \widehat{\otimes} H_{2}}=\left(\varphi,\left(T_{1}^{*} \otimes T_{2}^{*}\right) \psi\right)_{H_{1} \widehat{\otimes} H_{2}}
$$

for all $\varphi \in D\left(T_{1} \otimes T_{2}\right)$ and all $\psi \in D\left(T_{1}^{*} \otimes T_{2}^{*}\right)$. 
(ii) Through induction it is of course possible to prove a corresponding result for the tensor product of $r$ operators $(r \in \mathbf{N})$, since the tensor product is associative.

Proof. A. By a direct calculation one easily verifies the relation

$$
\overline{T_{1}} \otimes \overline{T_{2}} \subset \overline{T_{1} \otimes T_{2}} .
$$

B. As the second step we shall consider the operator

$$
V:=T_{1}^{*} \overline{T_{1}} \otimes T_{2}^{*} \overline{T_{2}} .
$$

It is in $H_{1} \widehat{\otimes} H_{2}$ densely defined with the domain

$$
D(V)=D\left(T_{1}^{*} \overline{T_{1}}\right) \otimes D\left(T_{2}^{*} \overline{T_{2}}\right)
$$

and $V$ as a tensor product of two symmetric operators is symmetric in $H_{1} \widehat{\otimes} H_{2}$ (cf. [8], p. 9).

We shall now prove the relation

$$
V=T_{1}^{*} \overline{T_{1}} \otimes T_{2}^{*} \overline{T_{2}}=\left(T_{1}^{*} \otimes T_{2}^{*}\right)\left(\overline{T_{1}} \otimes \overline{T_{2}}\right) .
$$

Let us take two elements $u_{j} \in D\left(T_{j}^{*} \overline{T_{j}}\right)(j=1,2)$. We get

$$
\begin{aligned}
\left(T_{1}^{*} \overline{T_{1}} \otimes T_{2}^{*} \overline{T_{2}}\right) & \left(u_{1} \otimes u_{2}\right)=T_{1}^{*} \overline{T_{1}} u_{1} \otimes T_{2}^{*} \overline{T_{2}} u_{2} \\
& =\left(T_{1}^{*} \otimes T_{2}^{*}\right)\left(\overline{T_{1}} u_{1} \otimes \overline{T_{2}} u_{2}\right) \\
& =\left(T_{1}^{*} \otimes T_{2}^{*}\right)\left(\overline{T_{1}} \otimes \overline{T_{2}}\right)\left(u_{1} \otimes u_{2}\right) .
\end{aligned}
$$

Since the corresponding relation is valid also for all finite linear combinations of elements of the form $u_{1} \otimes u_{2}$, we get the relation (2.2).

C. Now we have to prove the statement that the operator

$$
W:=\left(\overline{T_{1}^{*} \otimes T_{2}^{*}}\right)\left(\overline{T_{1} \otimes T_{2}}\right)
$$

is symmetric. Since $\overline{T_{1} \otimes T_{2}}$ is densely defined and closed, the operator

$$
\left(T_{1} \otimes T_{2}\right)^{*}\left(\overline{T_{1} \otimes T_{2}}\right)
$$

is self-adjoint by Theorem 1.1. From

$$
\left(\overline{T_{1}^{*} \otimes T_{2}^{*}}\right)\left(\overline{T_{1} \otimes T_{2}}\right) \subset\left(T_{1} \otimes T_{2}\right)^{*}\left(\overline{T_{1} \otimes T_{2}}\right)
$$

the statement then follows. 
D. We will further prove that the operator $W:=\left(\overline{T_{1}^{*} \otimes T_{2}^{*}}\right)\left(\overline{T_{1} \otimes T_{2}}\right)$ is closed. Therefore we choose a sequence

$$
\left\{u_{k}\right\} \subset D\left(\left(\overline{T_{1}^{*} \otimes T_{2}^{*}}\right)\left(\overline{T_{1} \otimes T_{2}}\right)\right)
$$

with elements $u, f \in H_{1} \widehat{\otimes} H_{2}$ having the properties

$$
\left\|u_{k}-u\right\|_{H_{1} \widehat{\otimes} H_{2}} \rightarrow 0
$$

and

$$
\left\|\left(\overline{T_{1}^{*} \otimes T_{2}^{*}}\right)\left(\overline{T_{1} \otimes T_{2}}\right) u_{k}-f\right\|_{H_{1} \widehat{\otimes} H_{2}} \rightarrow 0 .
$$

By (2.1) it follows

$$
\left\|\left(\overline{T_{1} \otimes T_{2}}\right)\left(u_{k}-u_{l}\right)\right\|_{H_{1} \widehat{\otimes} H_{2}}^{2}=\left(\left(\overline{T_{1}^{*} \otimes T_{2}^{*}}\right)\left(\overline{T_{1} \otimes T_{2}}\right)\left(u_{k}-u_{l}\right), u_{k}-u_{l}\right)_{H_{1} \widehat{\otimes} H_{2}} \rightarrow 0 .
$$

This means that $\left\{\left(\overline{T_{1} \otimes T_{2}}\right) u_{k}\right\}_{k \in \mathbf{N}}$ is a Cauchy sequence in $H_{1} \widehat{\otimes} H_{2}$, and this sequence has a limit element $v \in H_{1} \widehat{\otimes} H_{2}$. Since the operators $\overline{T_{1} \otimes T_{2}}$ and $\overline{T_{1}^{*} \otimes T_{2}^{*}}$ are closed, we see that

$$
\left(\overline{T_{1} \otimes T_{2}}\right) u=v, \quad\left(\overline{T_{1}^{*} \otimes T_{2}^{*}}\right) v=f
$$

and, furthermore,

$$
\left(\overline{T_{1}^{*} \otimes T_{2}^{*}}\right)\left(\overline{T_{1} \otimes T_{2}}\right) u=f .
$$

Thus the operator $\left(\overline{T_{1}^{*} \otimes T_{2}^{*}}\right)\left(\overline{T_{1} \otimes T_{2}}\right)$ is closed.

E. Now we introduce two (densely defined) operators in $H_{1} \widehat{\otimes} H_{2}$ :

$$
\begin{aligned}
& A:=T_{1}^{*} \overline{T_{1}} \otimes I_{2} \quad \text { with the domain } D(A):=D(V), \\
& B:=I_{1} \otimes T_{2}^{*} \overline{T_{2}} \quad \text { with the domain } D(B):=D(V) .
\end{aligned}
$$

(We denote the identity operator of $H_{j}(j=1,2)$ by $I_{j}$ and the identity operator of $H:=H_{1} \widehat{\otimes} H_{2}$ by $I_{H}$.)

Let us note that $A$ is here defined as the restriction of the symmetric operator $T_{1}^{*} \overline{T_{1}} \otimes I_{2}$ with the maximal domain $D\left(T_{1}^{*} \overline{T_{1}}\right) \otimes H_{2}$ to the domain $D(V)$ of $V$, and $B$ is defined analogously. As restrictions of symmetric operators the operators $A$ and $B$ are symmetric on $H$.

F. The operators $A, B$ and $V$ fulfill for $u \in D(A)=D(B)=D(V)$ the relations

$$
(A u, u)_{H} \geq 0, \quad(B u, u)_{H} \geq 0, \quad(V u, u)_{H} \geq 0
$$


and

$$
(A u, V u)_{H} \geq 0, \quad(B u, V u)_{H} \geq 0 .
$$

Only the latter relation will proved here.

An element $u \in D(V)$ has a representation

$$
u=\sum_{r=1}^{m} u_{1, r} \otimes u_{2, r}
$$

with $u_{j, r} \in D\left(T_{j}^{*} \overline{T_{j}}\right)$. By the bilinearity of the scalar product one gets the following estimation:

$$
\begin{aligned}
(B u, V u)_{H} & =\left(\sum_{r=1}^{m} u_{1, r} \otimes T_{2}^{*} \overline{T_{2}} u_{2, r}, \sum_{s=1}^{m} T_{1}^{*} \overline{T_{1}} u_{1, s} \otimes T_{2}^{*} \overline{T_{2}} u_{2, s}\right)_{H} \\
& =\sum_{r, s=1}^{m}\left(u_{1, r} \otimes T_{2}^{*} \overline{T_{2}} u_{2, r}, T_{1}^{*} \overline{T_{1}} u_{1, s} \otimes T_{2}^{*} \overline{T_{2}} u_{2, s}\right)_{H} \\
& =\sum_{r, s=1}^{m}\left(u_{1, r}, T_{1}^{*} \overline{T_{1}} u_{1, s}\right)_{H_{1}}\left(T_{2}^{*} \overline{T_{2}} u_{2, r}, T_{2}^{*} \overline{T_{2}} u_{2, s}\right)_{H_{2}} \\
& =\sum_{r, s=1}^{m}\left(\overline{T_{1}} u_{1, r}, \overline{T_{1}} u_{1, s}\right)_{H_{1}}\left(T_{2}^{*} \overline{T_{2}} u_{2, r}, T_{2}^{*} \overline{T_{2}} u_{2, s}\right)_{H_{2}} \\
& =\sum_{r, s=1}^{m}\left(\overline{T_{1}} u_{1, r} \otimes T_{2}^{*} \overline{T_{2}} u_{2, r}, \overline{T_{1}} u_{1, s} \otimes T_{2}^{*} \overline{T_{2}} u_{2, s}\right)_{H} \\
& =\left(\sum_{r=1}^{m} \overline{T_{1}} u_{1, r} \otimes T_{2}^{*} \overline{T_{2}} u_{2, r}, \sum_{s=1}^{m} \overline{T_{1}} u_{1, s} \otimes T_{2}^{*} \overline{T_{2}} u_{2, s}\right)_{H} \\
& \geq 0 .
\end{aligned}
$$

G. For the symmetric operator

$$
S:=V+A+B+I_{H}
$$

with the domain $D(S):=D(V)$ the relations (2.3) imply

$$
(S u, u)_{H} \geq(u, u)_{H} \quad \text { for all } u \in D(S) .
$$

We will show here that

$$
\overline{R(S)}=H
$$


Let $h_{j}$ be an arbitrary element in $H_{j}(j=1,2)$. By Theorem 1.1 there exists an element $u_{j} \in D\left(T_{j}^{*} \overline{T_{j}}\right)$ with

$$
h_{j}=\left(T_{j}^{*} \overline{T_{j}}+I_{j}\right) u_{j}
$$

One further gets

$$
\begin{aligned}
h_{1} \otimes h_{2} & =\left(T_{1}^{*} \overline{T_{1}} u_{1}\right) \otimes\left(T_{2}^{*} \overline{T_{2}} u_{2}\right)+u_{1} \otimes\left(T_{2}^{*} \overline{T_{2}} u_{2}\right)+\left(T_{1}^{*} \overline{T_{1}} u_{1}\right) \otimes u_{2}+u_{1} \otimes u_{2} \\
& =S\left(u_{1} \otimes u_{2}\right) .
\end{aligned}
$$

As the completion of the linear hull of all elements $h_{1} \otimes h_{2}$ is the space $H$, we get the statement (2.6).

From (2.5) and (2.6) it follows that already

$$
R(\bar{S})=H .
$$

H. By the relations (2.3) and (2.4) we get for all $u \in D(V)$

$$
\begin{aligned}
\|S u\|_{H}\left\|\left(V+I_{H}\right) u\right\|_{H} & \geq\left|\left(S u,\left(V+I_{H}\right) u\right)_{H}\right| \\
& =(V u+A u+B u+u, V u+u)_{H} \\
& \geq\left\|\left(V+I_{H}\right) u\right\|_{H}^{2}
\end{aligned}
$$

and furthermore

$$
\|S u\|_{H} \geq\left\|\left(V+I_{H}\right) u\right\|_{H} .
$$

The last relation implies

$$
D(\bar{S}) \subset D\left(\overline{V+I_{H}}\right) .
$$

I. It follows from part $\mathrm{F}$ of this proof that the symmetric operator $V+I_{H}$ fulfills the relation

$$
\left(\left(V+I_{H}\right) u, u\right)_{H} \geq\|u\|_{H}^{2}
$$

for all $u \in D(V)$. We shall show that

$$
\overline{R\left(V+I_{H}\right)}=H \text {. }
$$

Let $z \in H$ be an element with

$$
\left(z,\left(V+I_{H}\right) u\right)_{H}=0 \quad \text { for all } u \in D(V) .
$$

By (2.7) there exists an element $v \in D(\bar{S}) \subset D\left(\overline{V+I_{H}}\right)$ with $z=\bar{S} v$. The relation (2.10) implies

$$
\left(\bar{S} v,\left(\overline{V+I_{H}}\right) v\right)_{H}=0 .
$$

Since (2.8) is valid also for the closures $\bar{S}$ and $\overline{V+I_{H}}$, we get

$$
\left(\overline{V+I_{H}}\right) v=0 \text {. }
$$

Finally, the relation (2.9) implies $v=0$. Thus the element $z \in H$ must be the zero element, and the relation $\overline{R\left(V+I_{H}\right)}=H$ is proved. 
J. Since we have

$$
\left(V+I_{H}\right) u=\left(T_{1}^{*} \overline{T_{1}} \otimes T_{2}^{*} \overline{T_{2}}+I_{H}\right) u
$$

for $u \in D(V)$, we can conclude that the range

$$
R\left(T_{1}^{*} \overline{T_{1}} \otimes T_{2}^{*} \overline{T_{2}}+I_{H}\right)=R\left(\left(T_{1}^{*} \otimes T_{2}^{*}\right)\left(\overline{T_{1}} \otimes \overline{T_{2}}\right)+I_{H}\right)
$$

is dense in the space $H$.

K. Now we can apply Corollary 1.2 to the operator

$$
\overline{T_{1}} \otimes \overline{T_{2}}
$$

and we get the essential maximality of $\overline{T_{1}} \otimes \overline{T_{2}}$ in view of $T_{1}^{*} \otimes T_{2}^{*}$; because $\overline{\overline{T_{1}} \otimes \overline{T_{2}}}=\overline{T_{1} \otimes T_{2}}$, Theorem 2.1 is proved.

Finally, let us remark that the above proof could be somewhat shorter if we had used the well-known corresponding result for self-adjoint operators.

\section{The essential maximality of linear partial differential operators}

Let $G$ be an arbitrary domain in $\mathbf{R}^{n}$. We now consider in $G$ a linear partial differential operator

$$
L(\cdot, D)=\sum_{|\alpha| \leq m} a_{\alpha}(\cdot) D^{\alpha}
$$

of order $m \in \mathbf{N}$. Here we have defined $D:=\left(D_{1}, \ldots, D_{n}\right)$ with $D_{j}=-i \partial / \partial x_{j}$ and $D^{\alpha}:=D_{1}^{\alpha_{1}} \cdots D_{n}^{\alpha_{n}}$ for $\alpha:=\left(\alpha_{1}, \ldots, \alpha_{n}\right) \in \mathbf{N}_{0}^{n}=(\mathbf{N} \cup\{0\})^{n}$ and $|\alpha|:=$ $\alpha_{1}+\cdots+\alpha_{n}$. We suppose that, for the complex-valued coefficient functions $a_{\alpha}$, all derivatives $D^{\nu} a_{\alpha}$ for $\nu \in \mathbf{N}_{0}^{n}$ with $\nu \leq \alpha$ (i.e. for all $\nu=\left(\nu_{1}, \ldots, \nu_{n}\right) \in \mathbf{N}_{0}^{n}$ with $\left.\nu_{1} \leq \alpha_{1}, \ldots, \nu_{n} \leq \alpha_{n}\right)$ are continuous in $G$. On this condition we have for the formal adjoint linear partial differential operator $L^{\prime}(\cdot, D)$ defined through the equation

$$
(L(\cdot, D) \varphi, \psi)_{0}=\left(\varphi, L^{\prime}(\cdot, D) \psi\right)_{0} \quad \text { for all } \varphi, \psi \in C_{0}^{\infty}(G)
$$

(with the $L^{2}(G)$ scalar product $\left.(\cdot, \cdot)_{0}\right)$ the expression

$$
\begin{aligned}
L^{\prime}(x, D) \psi(x) & =\sum_{|\alpha| \leq m} D^{\alpha}\left(\overline{a_{\alpha}(x)} \psi(x)\right) \\
& =\sum_{|\alpha| \leq m} \sum_{\nu \leq \alpha}\left(\begin{array}{l}
\alpha \\
\nu
\end{array}\right)\left(D^{\nu} \overline{a_{\alpha}(x)} D^{\alpha-\nu} \psi(x)\right) \quad \text { for } \psi \in C_{0}^{\infty}(G),
\end{aligned}
$$


where

$$
\left(\begin{array}{l}
\alpha \\
\nu
\end{array}\right):=\left(\begin{array}{c}
\alpha_{1} \\
\nu_{1}
\end{array}\right) \cdots\left(\begin{array}{c}
\alpha_{n} \\
\nu_{n}
\end{array}\right) \text {. }
$$

The differential operators $L(\cdot, D)$ and $L^{\prime}(\cdot, D)$ induce in the Hilbert space $L^{2}(G)$ the linear operators $L$ and $L^{\prime}$ defined by

$$
\begin{aligned}
D(L) & :=C_{0}^{\infty}(G), & & D\left(L^{\prime}\right):=C_{0}^{\infty}(G), \\
L \varphi & :=L(\cdot, D) \varphi & & \text { for } \varphi \in D(L), \\
L^{\prime} \psi & :=L^{\prime}(\cdot, D) \psi & & \text { for } \psi \in D\left(L^{\prime}\right) .
\end{aligned}
$$

The operators $L$ and $L^{\prime}$ are closable as in the abstract setting in 1 . In the literature normally the closures $\bar{L}$ and $\overline{L^{\prime}}$ of $L$ and $L^{\prime}$ are called the minimal realizations of $L(\cdot, D)$ and $L^{\prime}(\cdot, D)$, and the adjoints $L^{\prime *}$ and $L^{*}$ of $L^{\prime}$ and $L$ the maximal realizations of $L(\cdot, D)$ and $L^{\prime}(\cdot, D)$ (namely, e.g. $L \subset \bar{L} \subset L^{\prime *}$ ).

Since in our present case the domain $D\left(L^{\prime}\right)$ of the operator $L^{\prime}$ coincides with the domain $D(L)$ of $L$ in a canonical way, we do not need the words "in view of the operator $L^{\prime}$ " (which we needed in the abstract setting) in the following definition of the essential maximality of the differential operator $L(\cdot, D)$ (cf. e.g. $[2],[4],[6])$.

Definition 3.1. The differential operator $L(\cdot, D)$ is called essentially maximal if the relation $L^{*}=\overline{L^{\prime}}$ is valid.

We now have the following results as corollaries of Theorem 1.2 and of Theorem 2.1:

Theorem 3.2. For the essential maximality of the differential operator $L(\cdot, D)$ it is necessary and sufficient that the range of the operator $\overline{L^{\prime}} \bar{L}+I$ is the whole space $L^{2}(G)$.

Theorem 3.3. Let $G_{j} \subset \mathbf{R}^{n_{j}}(1 \leq j \leq r, r \in \mathbf{N})$ be domains in $\mathbf{R}^{n_{j}}$. The linear partial differential operators

$$
L_{j}(\cdot, D)=\sum_{\left|\alpha^{j}\right| \leq m_{j}} a_{\alpha^{j}}(\cdot) D^{\alpha^{j}}, \quad \alpha^{j} \in \mathrm{N}_{0}^{n_{j}}, 1 \leq j \leq r
$$

are defined in the domains $G_{j}$. We suppose that the derivatives $D^{\nu^{j}} a_{\alpha^{j}}\left(x^{j}\right)$ for all $\nu^{j} \in \mathbf{N}_{0}^{n_{j}}$ with $\nu^{j} \leq \alpha^{j}$ exist and are continuous functions in $G_{j}$.

Then also the formal adjoint differential operators $L_{j}^{\prime}(\cdot, D)$ are defined.

We further suppose that the differential operators $L_{j}(\cdot, D)$ are essentially maximal in $L^{2}\left(G_{j}\right)$.

Then the product operator

$$
L\left(x^{1}, \ldots, x^{r}, D\right)=\sum_{\left|\alpha^{1}\right| \leq m_{1}, \ldots,\left|\alpha^{r}\right| \leq m_{r}} a_{\alpha^{1}}\left(x^{1}\right) \cdots a_{\alpha^{r}}\left(x^{r}\right) D^{\left(\alpha^{1}, \ldots, \alpha^{r}\right)}
$$


On the essential maximality of linear operators in a Hilbert space

is essentially maximal in $L^{2}\left(G_{1} \times \cdots \times G_{r}\right)$. (For the differential operator $L\left(x^{1}, \ldots, x^{r}, D\right)$ the formal adjoint operator $L^{\prime}\left(x^{1}, \ldots, x^{r}, D\right)$ is defined in $G_{1} \times \cdots \times G_{r}$.)

Proof. By Theorem 2.1 we get

$$
\left(L_{1} \otimes \cdots \otimes L_{r}\right)^{*}=\overline{L_{1}^{*} \otimes \cdots \otimes L_{r}^{*}}
$$

and, since $L_{j}(\cdot, D)$ is essentially maximal, also

$$
\begin{aligned}
& =\overline{\overline{L^{\prime}}{ }_{1} \otimes \cdots \otimes \overline{L_{r}^{\prime}}} \\
& =\overline{{\overline{L_{1}^{\prime}} \otimes \cdots \otimes L_{r}^{\prime}}^{\prime}} \\
& =\overline{\left(L_{1} \otimes \cdots \otimes L_{r}\right)^{\prime}} .
\end{aligned}
$$

(Here we used the fact that the closure of the tensor product of closures of operators coincides with the closure of the tensor product of operators and that the tensor product of formal adjoints coincides with the formal adjoint of the tensor product of the original operators.) 


\section{Bibliography}

[1] Goldstein, R.A.: Equality of minimal and maximal extensions of partial differential operators in $L_{p}\left(R^{n}\right)$. - Proc. Amer. Math. Soc. 17, 1966, 1031-1033.

[2] HEss, P.: Über die wesentliche Maximalität gleichmäßig stark elliptischer Operatoren in $L^{2}\left(R^{n}\right)$. - Math. Z. 107, 1968, 67-70.

[3] Kalf, H., U.-W. Schmincke, J. Walter, and R. Wüst: On the spectral theory of Schrödinger and Dirac operators with strongly singular potentials. - Spectral theory and differential equations, Proceedings of the Symposium held at Dundee, Scotland, 1-19 July, 1974. Lecture Notes in Mathematics 448, 182-226. Springer-Verlag, Berlin-Heidelberg-New York, 1975.

[4] LouhivaARA, I.S., and C.G. Simader: Über nichtelliptische lineare partielle Differentialoperatoren mit konstanten Koeffizienten. - Ann. Acad. Sci. Fenn. Ser. A I Math. 513, 1972, 1-22.

[5] Riesz, F., and B. Sz.-NagY: Vorlesungen über Funktionalanalysis. - [Vierte, durchgesehene Auflage.] Hochschulbücher für Mathematik 27. Deutscher Verlag der Wissenschaften, Berlin, 1982.

[6] Steinig, B.: Remarks on the essential maximality of linear partial differential operators. - Inauguraldissertation. Freie Universität Berlin, Fachbereich Mathematik, Berlin, 1989.

[7] Tervo, J.: Zur Theorie der koerzitiven linearen partiellen Differentialoperatoren. - Ann. Acad. Sci. Fenn. Ser. A I Math. Dissertationes 45, 1983.

[8] Vala, K.: Sur le produit tensoriel des espaces hilbertiens. - Ann. Acad. Sci. Fenn. Ser. A I Math. 267, 1959.

[9] WeidmanN, J.: Linear operators in Hilbert spaces. - Graduate Texts in Mathematics 68. Springer-Verlag, New York-Heidelberg-Berlin, 1980.

Freie Universität Berlin

Institut für Mathematik I

Arnimallee 2-6

DW-1000 Berlin 33

Bundesrepublik Deutschland

Received 6 August 1990 